\title{
Assessing multiple object tracking in young children using a game
}

\author{
Kimiko Ryokai · Faraz Farzin · Eric Kaltman • Greg Niemeyer
}

Published online: 7 March 2013

(c) The Author(s) 2013. This article is published with open access at Springerlink.com

\begin{abstract}
Visual tracking of multiple objects in a complex scene is a critical survival skill. When we attempt to safely cross a busy street, follow a ball's position during a sporting event, or monitor children in a busy playground, we rely on our brain's capacity to selectively attend to and track the position of specific objects in a dynamic scene. This ability to visually track simultaneously moving objects in a continuously changing and multisensory environment is a critical component of nearly all forms of visual-motor coordination. While methods for assessing Multiple Object Tracking (MOT) in adults are well established, due to challenges associated with designing a MOT task suitable for young children, we have little understanding of MOT abilities under the age of 5 years. To better understand how and when young children learn to track multiple objects, we designed, implemented and evaluated TrackFX, the first game-based MOT task running on a touch tablet designed for children as young as 30 months old. We present findings from an empirical study of 31 children between the age of 30 and 58 months and implications for game-based learning.
\end{abstract}

Keywords Visual-spatial skills · Attention · Toddlers · Game · Touch tablet · Mobile technology

K. Ryokai (ه)

School of Information, University of California, Berkeley, Berkeley, CA 94720-1740, USA

e-mail: kimiko@ischool.berkeley.edu

F. Farzin

Department of Psychology, Stanford University, Stanford, CA 94305, USA

e-mail: ffarzin@stanford.edu

E. Kaltman

Department of Computer Science, University of California, Santa Cruz, Santa Cruz, CA 95064, USA e-mail: ekaltman@gmail.com

G. Niemeyer

Art Practice, University of California, Berkeley, Berkeley, CA 94720-1740, USA

e-mail: gregniemeyer@gmail.com 


\section{Introduction}

We live in a physically dynamic world. When picking a safe time to cross a busy street or to change lanes while driving, when assessing the ball's position while playing a sport, or when monitoring children in a busy playground, the brain is faced with the demanding task of selecting, attending to, and tracking the position of objects in a dynamic scene. The ability to visually track simultaneously moving objects in a continuously changing and multisensory environment is challenging but critical for human survival as it is essential for nearly all forms of visual-motor coordination.

To study the complex mental processes underlying object tracking, a controlled laboratory task called the Multiple Object Tracking (MOT) task was created (Pylyshyn and Storm 1988) and has been used successfully for over 20 years in the discipline of cognitive psychology to study adults' tracking performance. Among the general population, an adult can typically track up to four simultaneously moving objects. This illustrates that there is a significant capacity limitation in selective visual attention. For example, if there are 5 players on a field, each running around in a different pattern, we could successfully keep track of 4 of the players' movement simultaneously, but we may "lose track" of where the 5 th person is on the field. To track player 5 we would need to switch our attention to select him/her, rendering another player temporarily irrelevant information for tracking.

Recently, increased attention has been placed on studying how MOT skills develop during early childhood; specifically, when the ability to track moving objects emerges, and when it becomes adult-like. By studying MOT during childhood, it is thought that we will not only gain information about changes in object tracking performance with age, but we will also learn about the development of a variety of other cognitive functions (attention selection, information monitoring, and response inhibition) that are tapped during MOT. Additionally, understanding the development of MOT skills will shed light on the maturation of visual-motor abilities that depend on mechanisms involved in MOT.

Empirical findings have established that object tracking performance improves dramatically between childhood and young adulthood and have guided the proposal of a relationship between tracking and enumeration (Trick et al. 2003) and between tracking and attentional inhibition (Kipp 2005) in childhood. Both of these skills are thought to be correlated with MOT. In addition, findings from studies of children with a history of playing visual-spatial games (both virtual and physical) reveal that MOT skills are impacted by experience and thereby may be teachable (Trick et al. 2005). On the other hand, there is also evidence of clinical populations in which MOT performance is observed to be different from typically developing groups during childhood. Individuals with genetic disorders such as Williams syndrome, Turner's syndrome, Fragile X syndrome, and Autism Spectrum Disorders (ASD) have been found to show impairments in object tracking, typically in the form of a lower limit of successfully tracked objects (Farzin and Rivera 2010; Beaton et al. 2010; O'Hearn et al. 2005, 2010, 2011; Hahler et al. 2010). Results from this clinical developmental research suggest that tests of MOT performance could serve as a screening measure for specific forms of developmental delay.

There is value in understanding the early emergence of MOT in typically developing children, yet doing so involves overcoming a number of challenges. The biggest issue is creating an effective way of engaging very young children in MOT activities, but it is also critical that the task be scientifically valid (making it comparable to existing tasks used in the literature) and that the interface be able to support young children's independent interaction with the task. For example, MOT tasks require the ability to distinguish between identical moving items based on memory, a period of extended concentration and 
attention, and motor skills for interacting with the game, so if young children do not understand the rules, cannot physically engage with, or do not find the MOT task fun, we will not be able to measure their true tracking abilities. There is also a practical benefit in designing an MOT activity that can survive beyond experimental environments and can engage children over an extended period of time, which is that it can inform us of learning or training effects resulting from practice of the MOT task. Empirical research on MOT development has focused exclusively on children over the age of five in experimental settings such as research labs (Trick et al. 2003, 2005; O'Hearn et al. 2005) with the exception of O'Hearn et al. (2010). Outside of a laboratory setting with trained researchers, there are no MOT tasks designed for young children to "play" on their own. Is it possible to create an MOT task that young children can engage in as if they are playing a game by themselves, outside of the environment of a scientific research study?

Here, we transformed the classic lab-based MOT task into a game designed specifically for children as young as 3 years and we investigated MOT abilities using the game in a non-experimental setting. We designed and implemented TrackFX, the first game-based MOT task running on a touch tablet. While this work contributes to the disciplines of developmental and cognitive psychology, it is also a timely topic for research communities concerned with interactive learning media and children. With more and more young children today spending their "screen time" interacting with touch-based tablets and mobile phones, this work uncovers some of the elements that might impact the refinement of visual-spatial skill as a result of gameplay. Our questions were as follows: Can young children under the age of 5 interact with digital objects on a touch-tablet to accomplish a visual-motor task such as MOT? Would young children's tracking ability improve over time with more game play? We believe these key research questions are of interest to researchers and designers of interactive learning media for children.

We present our design of TrackFX, an empirical study of 31 children between the age of 30 and 58 months, results of our study, and we discuss implications for game-based learning.

\section{Multiple object tracking}

The classic experimental paradigm used to study the maintenance of object representations over changes in space and time is called MOT (Pylyshyn and Storm 1988). In the MOT task the observer is initially introduced to a number of identical objects, a subset of which are temporarily designated as targets, using a visual cue and then returning to their original (identical) form. All objects begin to move randomly and independently and when they stop moving 5-20 s later observers are asked to identify the targets. MOT then, is a measure of selective visual attention. In other words, the ability to select information (objects) at multiple locations that is relevant, while ignoring irrelevant information. The purpose of selective attention is to prevent sensory overload in the face of the overwhelming amount of visual information we receive and are required to process.

Over 20 years of research using MOT has characterized adult performance on the task, establishing the limits of items, space, and time that can be tracked (Pylyshyn and Storm 1988; Pylyshyn 2000; Alvarez and Franconeri 2007; Franconeri et al. 2010; Scholl 2009). This work suggests that adult tracking likely follows a normal distribution with a mean of about four objects, and that tracking is sensitive to changes in parameters such as number of objects, speed, and object occlusion. Importantly, the perceptual and cognitive skills implicated in the laboratory MOT task closely mimic skills employed in real-world 
everyday tasks including crossing a busy street, driving, etc.; namely, keeping track of what went where over time. Therefore, being better at MOT could facilitate other activities requiring tracking of highly dynamic visual-spatial content. Recent studies with action video game players and radar operators have revealed that an adults' tracking limit can change as a result of visual experiences, and therefore improves with extensive practice (Green and Bavelier 2003, 2006a, b; Barker et al. 2010).

When do children first learn to track multiple objects? Far less is known about when the skills needed to perform the MOT task initially develop and how these skills improve with experience. However, based on the research with adults, there is reason to believe that children may also learn based on their environments. For example, those children whose parents reported hours of action game play or action sport play performed significantly better on spatial tasks (Subrahmanyam and Greenfield 1994; Uttal et al. 2012). This provides some evidence that the skills involved in MOT may be trainable.

Recent studies have shown that typically developing children show increases in the number of objects they can track or remember between the ages of 3 and 6 . In a single study that included 40-60-month-olds (3-4-year-olds), the youngest age tested in an experimental setting thus far, (O'Hearn et al. 2010) found that children could track between 1 and 2 targets among a fixed number of 8 objects on the screen for a duration of $6 \mathrm{~s}$. While O'Hearn et al.'s study involved children as young as 40 months, the visual task used in the study was not designed as a stand-alone-game. The experimenter needed to provide detailed step-by-step verbal instructions to the children. While the children pointed at the targets on the computer screen, neither software nor hardware was designed to take response input directly from the children. Therefore, the adult experimenter who closely observed the child's gestures had to use the mouse to click on the object the children pointed in order to simulate the child's input. To date, empirical research on MOT development involves children over 40 months of age in experimental settings without a stand-alone game.

\section{Children's spatial skills and computer games}

Outside of controlled laboratory tasks, could playing computer games foster young children's cognitive skills? For example, McClurg and Chaillé (1987) found that playing select computer games for 5 min, twice a week, for 6 weeks improved fifth, seventh, and ninth graders' performance on a paper and pencil mental-rotation task in which students viewed a three-dimensional target shape in one orientation and indicated whether another shape is different or the same shape in a different orientation. In addition, fifth graders who had received the video game training scored higher than ninth graders who had not played the video games.

While it is often assumed that boys are better at spatial tasks than girls, computer games seem to improve spatial skills for girls and boys equally. Subrahmanyam and Greenfield (1994) and De Lisi and Wolford (2002) found that video game practice or computer-based instructional activity could significantly improve spatial skills for both girls and boys.

Do skills in computer games transfer to real world? Dunbar et al. have suggested that the attentional skills of children are linked with their behavior as pedestrians (Dunbar et al. 2001). They tested the ability of children between age 4 and 10 to switch visual attention and concentrate using a simple video game (to catch the frog which hops and hides behind the house and the train appearing and disappearing on the screen) and also observed the same subset of children crossing roads. Children who were better at the game, and thus 
more able to effectively switch attention visually, were more likely to show awareness of traffic when about to cross a road and crossed the road in an overall safer manner.

These studies suggest that computer game play can enhance spatial reasoning skills in children and that such skills can extend to the real world. We predict that engaging in games may have a positive cognitive effect for young children. However, the effect of game play on young children under the age of 4 has been less studied.

\section{Educational games}

The use of computer games in educational settings dates back to the 1950's (Cullingford et al. 1979). Since then, computer games have inspired a new generation of educators and game designers to consider the learning potential of games (e.g., Habgood and Overmars 2006; Gee 2003; Bowman 1982). Research into quantifying the learning benefits of games in educational settings is, however, still in its infancy. Nonetheless, teachers, parents, and policy makers recognize that games can support valuable skill development (Becker 2007; Dede 2005; van Eck 2006). Much of the drive to develop games for education comes from the observation that games increase students' internal motivations through the inclusion of aspects such as challenge, control, fantasy, and curiosity, while interpersonal motivations can be added through factors such as competition, cooperation, and recognition (Malone and Lepper 1987).

Well-designed educational games are simultaneously challenging and engaging for the players. Gredler (2004) suggested 5 design criteria for effective educational games: (1) winning should be based only on the demonstration of knowledge or skills. (2) the game should address important concepts or content. (3) the dynamics of the game should be easy to understand and interesting for the players but not obstruct or distort learning. (4) students should not lose points for wrong answers (they simply do not advance in the game), and (5) games should not be zero-sum exercises.

Educational games then require "a balance between the challenges perceived in a given situation and the skills a person brings to it," suggesting that "no activity can sustain it for long unless both the challenges and the skills become more complex" (Csikszentmihalyi 1988 , p. 30). Successful educational games teach the player primarily how to play the game and then how to be able to generalize to similar situations in real life (Gee 2007). From a cognitive perspective, the learning process through games can produce behavioral change by altering connections in the brain.

Our work builds on these design principles of educational games to design and develop a scientifically valid and engaging MOT game that may be useful in a classroom situation. TrackFX explicitly isolates a specific and important visual-spatial processing skill in a structured manner that increases or decreases the level of difficulty according to the player's skill level.

\section{Children and touch tablets}

Why consider MOT in the form of a video game? Could not MOT skills be fostered through physical sports and games such as soccer or dodgeball? While it is certainly possible to practice MOT-like skills in these traditional sports and games, they do not always allow us to isolate the impact of skills related specifically to visual-spatial tracking. For example, in a natural setting, busyness of the given background or the individual 
player's motor ability may influence one's tracking performance. A controlled virtual environment where the tracking task can be presented systematically with an appropriate difficulty level for the player may be an ideal platform for training MOT skills.

The hardware platform on which such MOT tasks are presented plays an important role in making the design usable and appropriate for young children. One of the age limiting factors of the MOT studies in the past has been the input device (e.g., a regular computer mouse is difficult for young children to use because their visual-spatial coordination is less refined than an older child). While young children's motor skills may be limited, more natural interfaces such as touch tablets are becoming available and seem to provide a better platform in support young children's MOT tasks. Designing touch tablet based games beneficial for young children's cognitive ability is a timely topic, as more young children are participating in screen time.

In late 2011, seven out of every 10 children under 12 in tablet-owning households used a tablet computer (Nielsen 2012). Seventy-seven percent of those surveyed said children play downloaded games on their tablets and $57 \%$ said children used tablets to access educational apps. While many young children play games with these new touch enabled mobile devices, few empirical studies have measured the cognitive benefit of these games.

One rare example is a study by Hourcade et al. (2011). They developed a variety of drawing and puzzle applications that run on multi-touch tablet and examined how children with ASD played with them. In their study with eleven 5- to 9-year old and ten 12- to 14-year-old children with ASD, they found that activities with these application increased pro-social behaviors such as collaboration and coordination, augmented appreciation for social activities, and provided children with novel forms of expression.

Children as young as 3 years old can use a touch tablet and a stylus. Couse and Chen (2010) studied young children between the ages of 3 and 6 years old learning to use the tablet as a medium for representing their ideas in various form of drawings. The children quickly learned to use and control the stylus on the touch pads, producing drawings that were sometimes even more advanced than what they are able to do with traditional pens and paper.

Some commercially available games for toddlers have been designed to run on touch tablets, such as iStartSmart ${ }^{\circledR}$ by Hatch. They claim to offer a series of emergent literacy and math games for toddlers and backend technology that track the learner's progress over time. However, they are not designed specifically to address MOT or other spatial cognition skills.

While the fields of Human Computer Interface and Interaction Design for Children have begun to investigate young children's use of tablets, more systematic studies of young children's ability to play games on tablets and the role of game play in children's cognitive ability for young children under the age of 5, are required. We investigate the design of a MOT game that is inviting and playable, and which also informs us about very young children's cognitive abilities.

\section{TrackFX design and implementation}

TrackFX is the first game to transfer the MOT paradigm, traditionally used with adults and children age 5 years and older, into a game that children under the age of 5 can engage in, in a non-experimental setting.

MOT tasks require a period of extended concentration and the ability to distinguish between identical moving items. To measure tracking abilities in a child it is important that 
the child is able to understand the basic rule of identifying the target object(s) and finds the MOT task engaging. In order to focus a child's attention on the screen for a sufficient amount of time, the visuals on the screen had to be inherently interesting for the young children to look at. While our game design was inspired by the original MOT task (Pylyshyn and Storm 1988), the visual theme needed to be adjusted for the younger children. After studying young children's books and consulting with the teachers at our testing site, we chose the theme of dancing ladybugs and spiders as most young children of this age in our society would be familiar with and could distinguish these bugs. We have also composed original music to go along with the animation so that the ladybugs look like they are happily dancing, in addition to other sound effects such as cheers that motivate the children to play successfully through the levels.

The game includes three element categories: leaves, ladybugs, and spiders. In each round, the player's goal is to track as many ladybugs as possible among a combination of ladybugs and spiders. Initially, $(n)$ identical leaves are introduced, randomly arranged on the screen. The leaves then rotate to reveal whether they are covering a ladybug (target) or a spider (distractor). After $4 \mathrm{~s}$, the leaves again hide the objects. The occluded objects then move randomly around the screen at a normalized velocity of $540 \mathrm{px} / \mathrm{sec}$ for $10 \mathrm{~s}$ before stopping. Once stationary, the player must correctly identify each target by selecting the leaf (leaves) covering the ladybug(s) (Fig. 1).

Every game is divided into a series of levels that maintain identical physics while adjusting the number of objects (targets and distractors). The first levels, 1-3, are introductory levels and are designed to facilitate player acclimation to the game. These introductory rounds are not included in the data analyses. Levels 1 and 2 have no leaves so that players can initially learn the identities of targets (ladybug) and distractors (spiders). After these levels, the occlusion mechanic (that of the leaves hiding and revealing the objects under occlusion) is demonstrated without any random movement on the screen. The first 3 levels of the game were built into make sure that the child understood the procedures and purpose of the game, and served as criteria for including data collected from gameplay on higher levels in analyses. Levels 4 and higher follow an identical structure as described above; occlusion, reveal, motion, and response (Fig. 2).

When the correct target is chosen by the player, the game reveals the target (ladybug) by moving away the occluder (leave), and the screen displays a "Congratulations!" message for $3 \mathrm{~s}$, and then the game moves on to the next level automatically. When an incorrect item is chosen, the game reveals the distractor (spider) briefly to indicate the incorrect choice, but the distractor is hidden again by the occluder, so that the player is given another chance to identify the correct target. The game repeats this process until the correct choice is made or until it runs out of the distractors.

Game levels were designed to proceed according to a psychophysical staircase procedure. The staircase was based on a 2-up-1-down decision rule such that if performance reached at or above $66 \%$ correct within a level twice in a row, the player advanced to the next level/stage, but if the player identified less than $66 \%$ of targets, the player dropped to the previous level/stage. A $66 \%$ correct performance level, or tracking threshold, was then estimated from average performance across the game session.

The game was designed to run on a mobile device such as a tablet. Most of the applications' development took advantage of the Adobe Flash Platform and its companion Air Framework, for development on the Samsung QXE1 Ultra-Mobile PC and Windows XP. The Flash platform allows for rapid prototyping of graphical applications.

The targets and distracters are each $50 \mathrm{px}$ in size on a $7^{\prime \prime}$ diagonal $(155 \mathrm{~mm} \times 86 \mathrm{~mm})$ with a resolution of $1,024 \times 600$ px. The clickable areas ("touch targets") for each target 


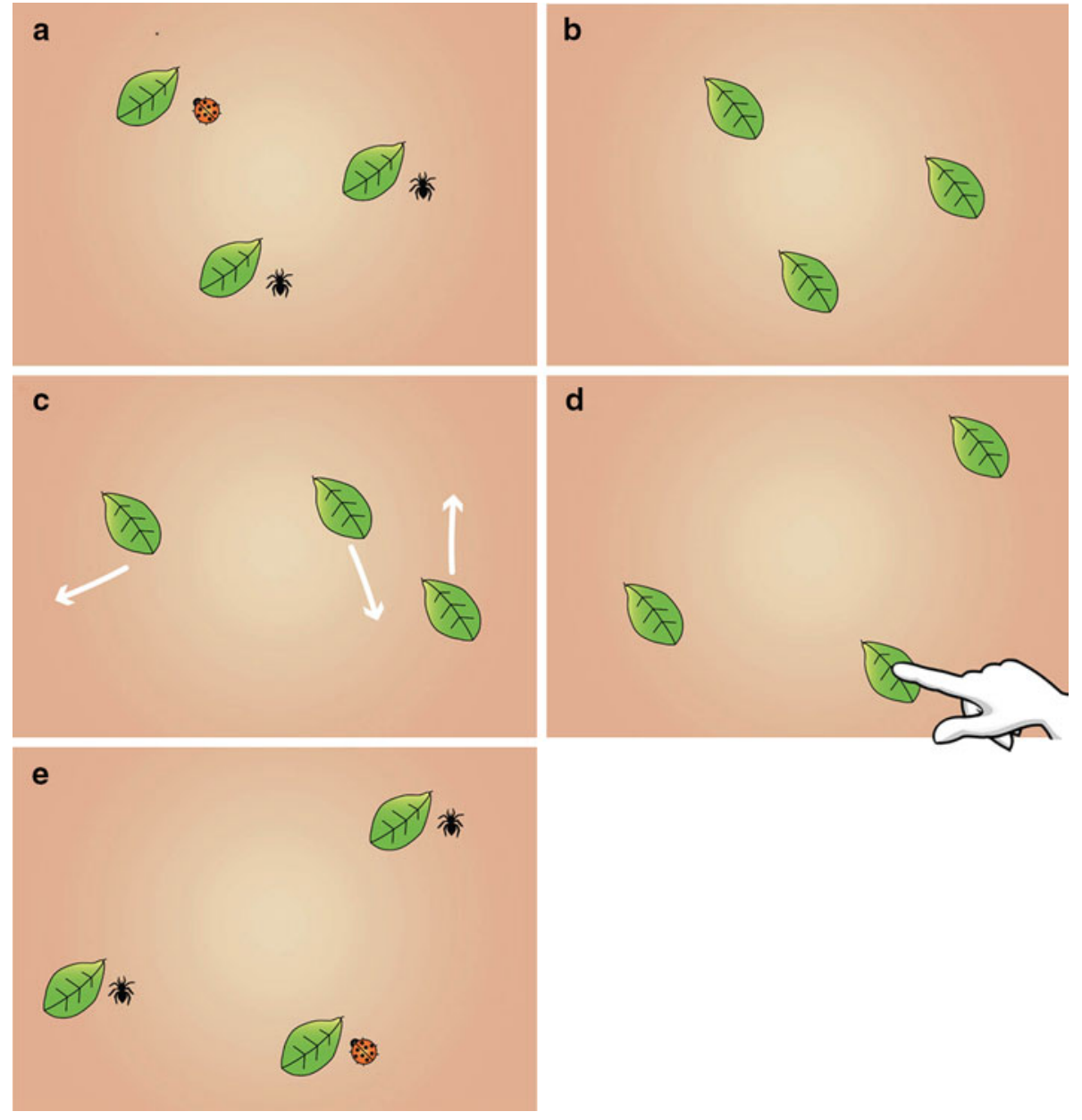

Fig. 1 a One target (ladybug) and two distractors (spiders) shown for 4 s. b The occluders (leaves) hide the target and distractors. All objects on the screen are now identical. c The occluded objects then move randomly around the screen at a normalized velocity of $540 \mathrm{px} / \mathrm{sec}$ for 10 seconds before stopping. d Once stationary, the player must correctly identify the target object by selecting the leaf covering the ladybug. e Once the player selects the correct target, the leaves turn to reveal the objects

and distractor are twice each object's visual size. This is necessary for two reasons: first, children had some difficulty accurately hitting the targets on the small UMPC screen, and second, each distractor leaf is twice the size of the target (100 px) and we wanted to ensure that every object had a similar bounding box. This box is necessary to assess collisions between the objects moving on screen and to enable the targets, distractors, and occluders to move in an identical fashion.

We also developed scripts to automatically generate simple statistics about each player, such as rounds completed, average threshold, etc. in CSV format, which could be opened by a statistical software package such as SPSS. 


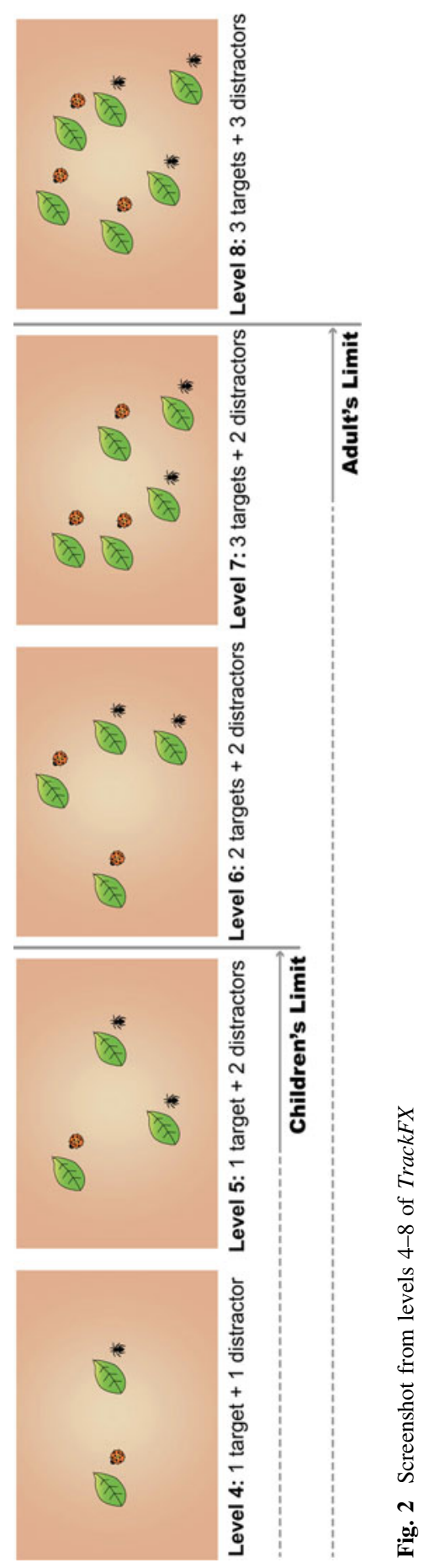




\section{Study methods}

Participants

Thirty-one children (16 female and 15 male) between the age of 30 and 58 months (Mean age $=47.65$ months, SD age $=6.39$ months), who were enrolled at the University of California, Berkeley, Harold E. Jones Child Study Center, participated in the study. Parental permission was obtained from all children prior to participation via a consent form. All children were tested at the Child Study Center by authors of the study.

Twelve adults between the age of 21 and 33 years (Mean age $=25.39$ years, SD age $=4.34$ years) also participated in the study. Adult participants naïve to the project were recruited through word-of-mouth, and were primarily students at the University of California, Berkeley. Adults played a procedurally identical version of the game as the children, except that the game was run from a web browser rather than on the tablet. Every asset and all sub system functions were the same on the web browser and on the tablet, but the size of the screen was larger in the web browser version. The motivation for testing a group of adults was to determine whether our game-based version of MOT replicated previous object tracking limits established from laboratory-based MOT tasks, thereby validating our measure.

\section{Procedure}

The testing was done in a room adjacent to the children's main classroom, during their regularly scheduled "choice time" activity period. The room provided familiarity for the children yet privacy from the main classroom activity. There was a maximum of three units present in the testing room and maximum of three children in the room at a time. Although there were up to three children in the testing room, each child was given his or her own device and was invited to play the game on his or her own pace at different corners of the room. Each child was also invited to play the game as long as they wished.

Testing was repeated across multiple days spanning 1-5 school days between tests. While we had originally planned to keep the frequency of exposure to the game consistent among children over time, it was difficult to control the duration between the tests, as children were absent due to illness or other scheduling conflicts.

There were two researchers present at any given time. On the first day, when the game was introduced to the children, the researcher introduced the basic rules of the game. "Follow the lady bug (demonstrating the child that s/he should follow it with their eyes and not with their fingers). Wait until it stops moving (showing the child not to touch the ladybug until it stops moving). Catch the ladybug, but not the spider (showing the child to touch the ladybug)."

Minimal training procedures were incorporated into the study design in order to allow children to become acquainted with the hardware and the goal of the game on their own. While each of our handheld devices was equipped with a touch screen, we decided to introduce a stylus as an input device to help the children point at the screen. This was due to the observation we made during the early pilot testing of the prototype system with young children. Originally, we had planned to have the children use their finger to touch the ladybugs on the touch screen, but during the pilot testing, the multi-touch screen system had difficulties isolating the input of the child's index finger from other parts of the child's hand that sometimes touched the screen simultaneously. For example, part of child's knuckle or palm could touch the multi-touch screen while the child tried to touch a specific 
location on the screen with his/her finger. When that happened, those other parts in contact with the screen were recognized as touch input instead of or in addition to the child's index finger. It was also difficult to instruct the young children to position their hands at a certain angle so as to not have any other parts of their hand except their index finger to touch the screen. After some observations, we found that having the stylus gave children much better control over their responses. While the children had to hold a stylus in their hand, we believe that having the stylus is much more natural and easier to control than having a computer mouse to do the same task.

Adults completed the consent process online and played the game from a web browser rather than on the tablet. The purpose was not to compare children with adults, rather it was to determine whether our game-based version of MOT replicated previous object tracking limits established from laboratory-based MOT tasks, thereby validating our measure.

\section{Results}

All 31 children successfully played TrackFX. Each child was able to respond using the handheld stylus and clearly understood the concept of the game, as evidenced by their successful performance on all baseline trials (first 3 levels). The children found the game engaging, playing on average 6 sessions (range $=1-17$ gameplays per child). We found no significant relationship between number of sessions played and age $(F(16,30)=0.577$, $p=0.854)$ or $\operatorname{sex} \mathrm{F}(1,29)=0.171, p=0.682)$, suggesting that the game was no more or less engaging to younger compared to older kids or to girls versus boys (Fig. 3).

Two measures served as indicators of object tracking performance: the proportion of correctly identified targets within each game level (expressed as $0-1$ ) and the overall tracking threshold (expressed as highest level of the game at which $66 \%$ of targets were correctly identified). Tracking thresholds provided a metric for the limit of object tracking for each game session in the sense that the number of targets present at the threshold level of the game was considered the tracking limit.

First we wanted to confirm whether adult performance matched the expected tracking limit based on previous research reports using the laboratory-based paradigm. To do so we evaluated adult tracking thresholds and found that, as a group, adults' tracking threshold was at level 7 (ranging between level 5 and level 9). Level 7 translates to being able to
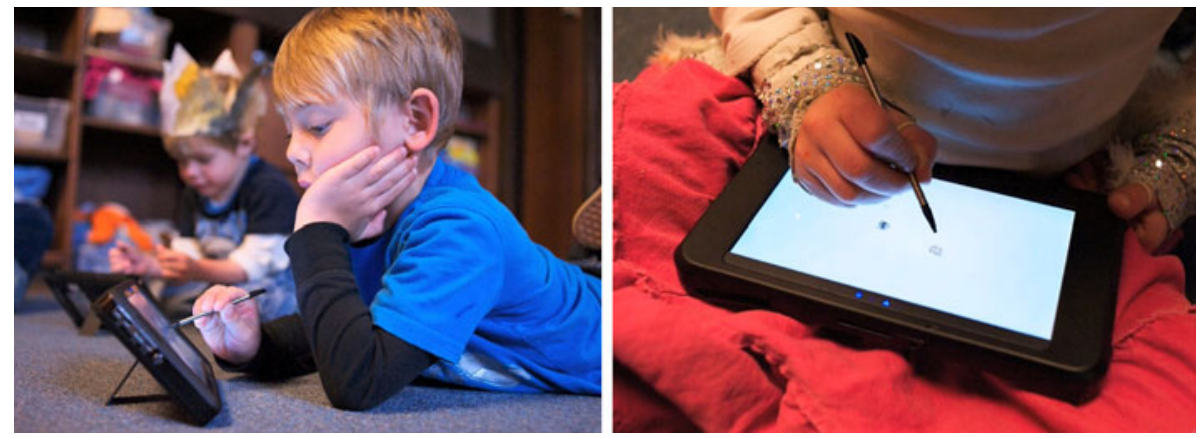

Fig. 3 Examples of children playing TrackFX. The tablet form factor allowed children to be in a variety of positions they found comfortable such as laying down on the floor (left) and sitting and with the tablet on their lap (right) 
track 3 targets and 2 distractors. Further, we found that performance was perfect up to level 5 and then declined with increasing game levels, and finally reached below $66 \%$ correct performance. These results are important because they validate that TrackFX is in fact measuring a cognitive ability that is consistent with MOT.

\section{Effect of level on tracking performance}

There was a direct impact of level on both adult's and children's ability to accurately identify targets. Figure 4 plots proportion correct versus level, and shows that all participants performed perfectly on levels 1,2 , and 3, in which an unoccluded target was static (level 1) or moving (level 2), or an occluded target was static (level 3). At level 4 the 2 groups deviate significantly, with children dropping to around 0.8 proportion of correctly identified targets. At each level beyond 4, children's performance was significantly lower than that of adults. Importantly, however, in both groups, the proportion of correctly identified targets dropped linearly with level. Specifically, in adults, the best fit linear regression of performance between level 5 and 10 produced a slope of -0.06 and an $R^{2}$ value of 0.87. In children, there was a linear drop between level 3 and level 9 with a best fit linear slope of -0.17 and an $R^{2}$ of 0.91 . Therefore, children's performance dropped more steeply as game level increased compared to that of adults.

This pattern of performance reveals a few key points about the game beyond the fact that higher levels were more difficult for all players. First, it tells us that the children had no difficulty seeing or discriminating the target from the distractor. Children also did not exhibit problems reporting the location of the target by touching the screen with the stylus. Finally, all children demonstrated a basic understanding that the goal of the game is to touch the ladybug and not the spider. In terms of game design, the nature of the difficulty curves obtained confirm that the dynamic leveling up technique is in fact adjusting the challenge or difficulty of the game for both groups of players, and is doing so in a nearly linear manner.

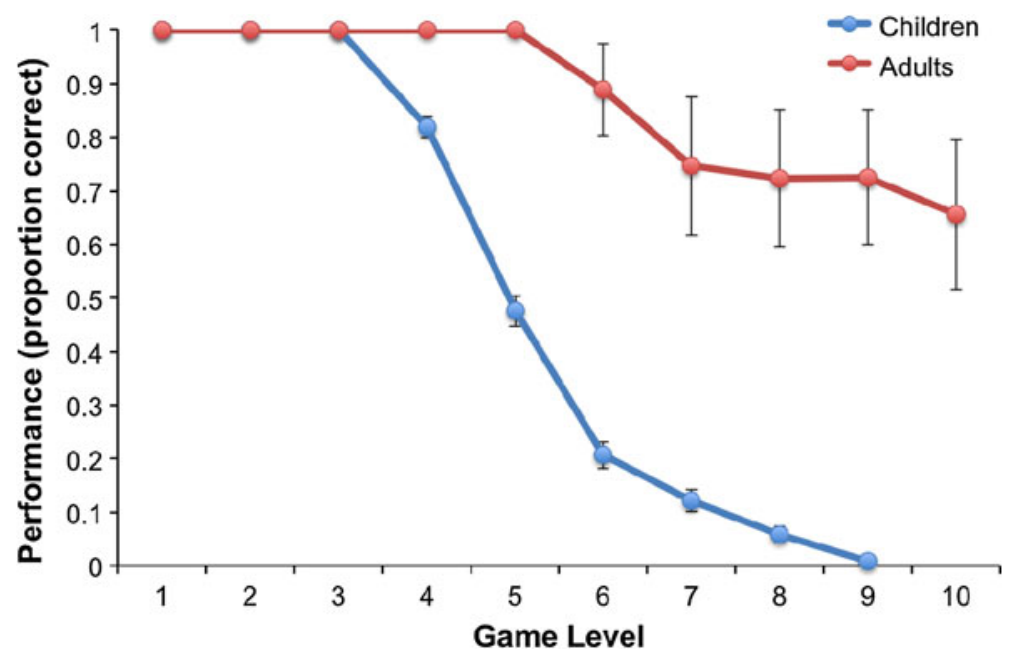

Fig. 4 Performance (proportion of correctly identified ladybugs) as a function of TrackFX level for children and adults. Error bars represent the standard errors of the mean 
As shown in Fig. 5, accuracy on the task was impacted both by target and distractor number, for both children and adults. However, adding distractors made the game substantially more difficult for children than adults, even when tracking a single target. For example, for children, tracking a single target with 2 versus 3 distractors on the screen, reduced performance by $20 \%$, but dropped performance by only $10 \%$ for adults. Similarly, tracking 3 targets generated a fixed performance level whether 2 or 3 distractors were present, but for children the presence of an additional distractor affected tracking performance by $6 \%$. This finding implies that additional objects on the screen-even if they were distractors and therefore should not have influenced target tracking-had negative consequences for children, but less so for adults. We interpret this as the employment of an attentional allocation strategy in adults that was not present in children. It is likely that for these combinations of items, adults are able to inhibit allocation of attentional resources to

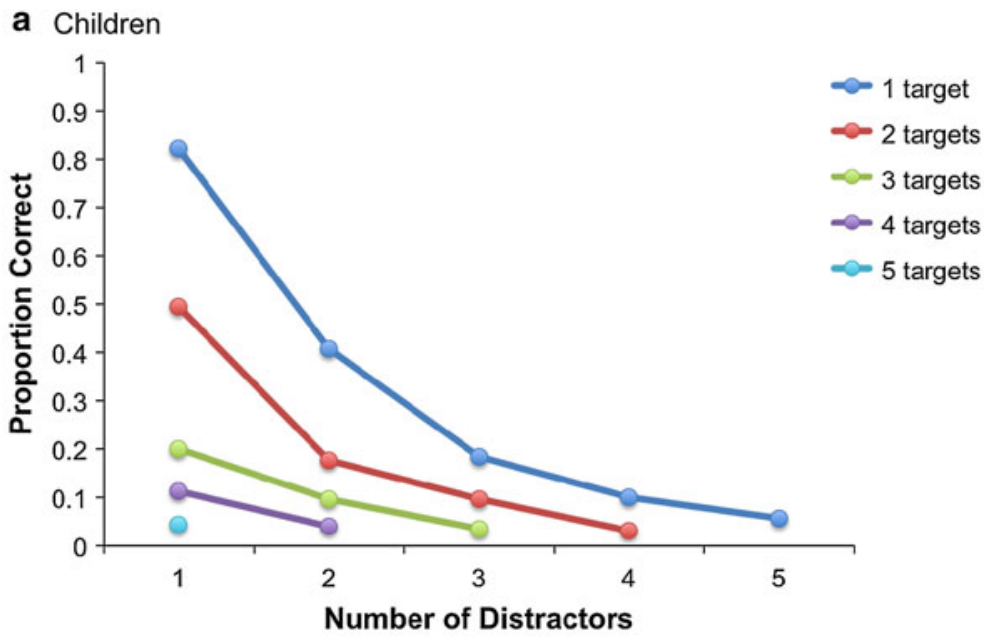

b Adults

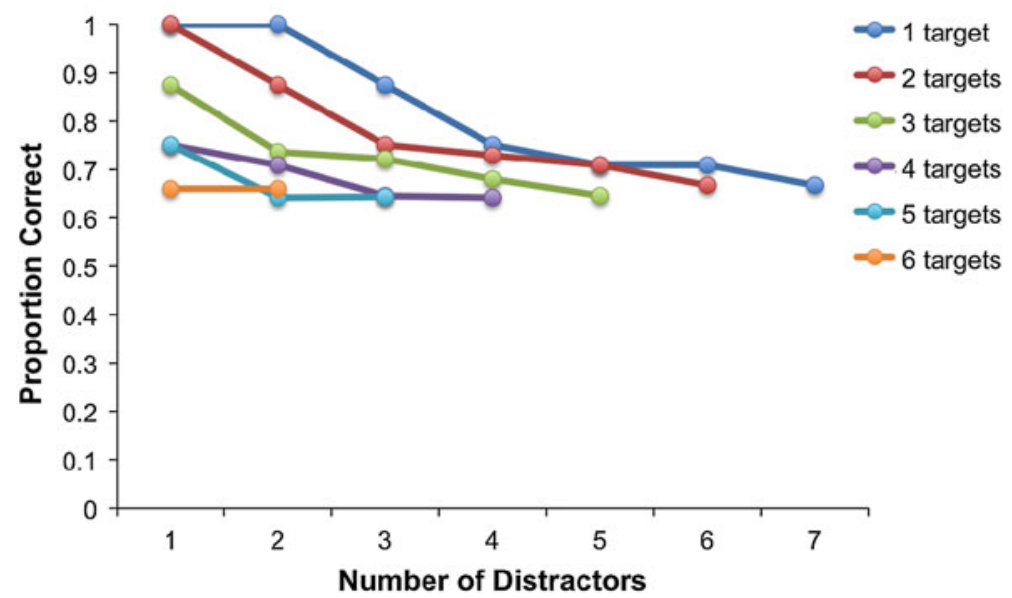

Fig. 5 Performance as a function of number of targets and number of distractors for $\mathbf{a}$ children and $\mathbf{b}$ adults 
the distractors, essentially ignoring distractors, in a way that children have not yet mastered.

Individual participants' tracking threshold level was obtained by averaging thresholds across all completed game sessions. The average threshold level across 31 children was Level 5 (Standard Error of Mean $(\mathrm{SEM})=0.15$; ranging from Level 4 to Level 7). Accordingly, the average number of targets tracked was 1, among 2 distractors (ranging from 1 target/1 distractor to 2.6 targets/4 distractors). We did not find a significant effect of age on threshold level $(\mathrm{F}(16,30)-0.654, p=0.794)$, however there was a slight trend for older children to achieve higher threshold levels (see Fig. 6). Also, a significantly positive correlation between the number of targets tracked and threshold obtained was found (Pearson correlation $=0.147, p=0.041$ ). On average children were able to track 1 target in the presence of 2 distractors.

To assess improvement in object tracking as a result of game play we evaluated tracking threshold as a function of play session by fitting a line to each participant's performance data and calculating the slope of the line. If an individual's ability to track objects improved over time with additional play sessions, the slope of the linear fit should be positive (greater than 0). If performance was unaffected by game play, the slope of the linear fit will be 0 . Thereby, we were able to identify trends of improvement for each child.

Thirty children played more than one session. Of these thirty, eleven $(37 \%)$ had performance slopes that were positive, with 2 children (both played 3 sessions) showing perfectly linear improvement over time with repeated gameplay (slope $=1$ ). However, we did not find a consistent learning effect over time for all 30 children. In fact, seventeen children showed negative performance slopes, however, these results were dominated by children who only played 2 sessions. Two children showed no change in performance across sessions. While this study took place at the children's day care center during their daily activities, distraction introduced by breaking the routine of their regularly scheduled classroom activities to play the game may have introduced variability in performance, especially during the first sessions as they adjusted to the novelty. Additionally, we observed that novelty of the game subsided across gameplay sessions, thereby increasing boredom and distractibility and resulting in lower tracking thresholds in the last play sessions. Therefore, we postulate that the negative slopes could be due to variable conditions of gameplay, rather than a direct result of the game.

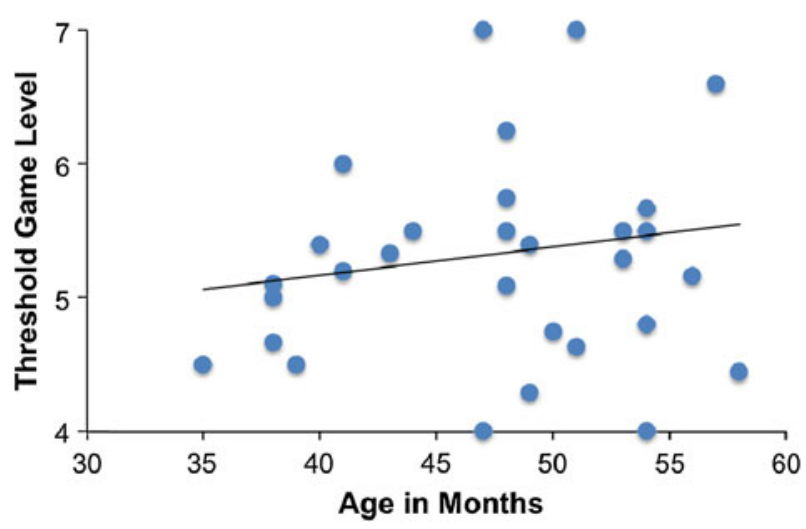

Fig. 6 Scatterplot of TrackFX threshold as a function of age effect of gameplay on tracking performance 


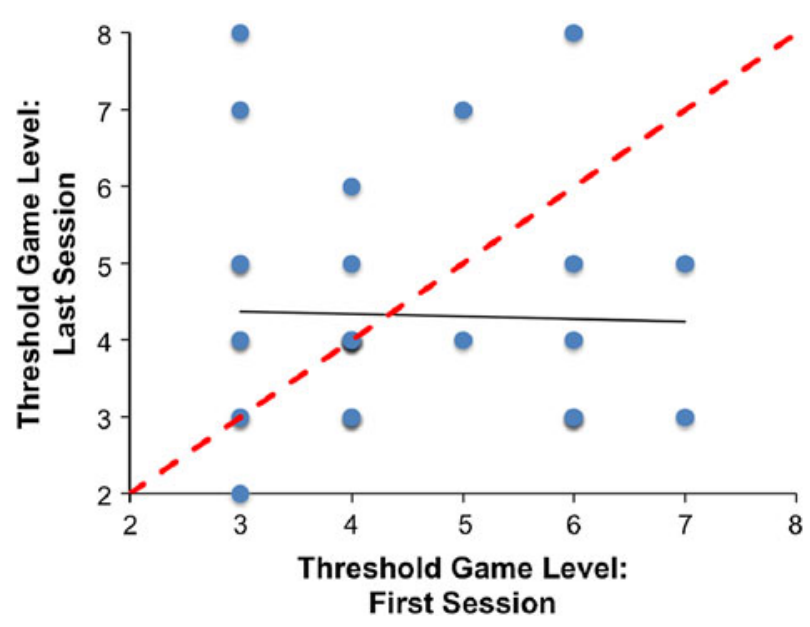

Fig. 7 Scatterplot of the first versus the last TrackFX game level reached for each child. Solid black line depicts the linear fit to the data. Dashed red line represents no change in gameplay, while points above line indicate improved play over time

While we found no difference in learning trends between girls and boys, there did appear to be a relationship between tracking improvement and number of gameplay sessions such that after six consecutive sessions, improvement reached an asymptotic level. Generally, no significant improvement was observed after the 6th gameplay. This could be related to the reduced and distracted attention mentioned above.

Figure 7 illustrates the individual variability in game performance between the first and the last gameplay session. Regression analysis on the threshold game level reached during the first versus the last session revealed an essentially flat, nonlinear relationship $(r=0.029 ; p=0.88)$ across the thirty children. Despite this nonsignificant learning effect, and in agreement with the slope fitting analysis described above, a select number of children did improve between the first and the last gameplay session. This can be seen in the points that fall above the dashed red line in Fig. 7.

\section{Conclusions}

To our knowledge, MOT performance has thus far only been measured within a research laboratory setting. Here, for the first time, we have successfully converted a lab-based MOT task into a game that children as young as 30 months old can play independently in a non-research setting. By introducing TrackFX in the real classrooms of toddlers and inviting the children to play with the game freestyle for as long as they wished during their regular daycare hours, we have demonstrated that it is possible to design a game that can be used to acquire valid scientific measures of MOT abilities while at the same time allowing the child to have fun in their natural environment.

The dynamic leveling up technique used in the design of TrackFX was effective in linearly adjusting the challenge or difficulty of the game for young children as well as adults. TrackFX produced a significant effect of level on tracking performance for children and adults and revealed that object tracking abilities are quite limited in very young children relative to adults. Children, on average, could track only 1 target among 2 distractors, uncovering the challenges young children face in selection and inhibition of visual 
attention in their dynamic world. Overall, while there was a large degree of variability in tracking performance within the group of children, we found that for some children there was a clear practice effect such that extended play improved tracking ability across test sessions. While further work is needed to confirm and optimize these learning effects, our study provides a first step towards empirically validating a cognitive task in a game format in a non-research setting.

There are a number of theoretical and practical implications of our findings. Within the context of perceptual and cognitive development theories, our measure of children's object tracking brings us closer to understanding how attentionally-demanding visual-spatial skills, and the regions of the brain that support these skills, mature with age. The limited tracking ability of 3-5-year-old children, relative to adults, as well as the marginally higher tracking limit ability in the older children within this age range, provide evidence that brain areas responsible for MOT develop and/or become maximally efficient with experience. Children's gameplay behavior can also be connected to performance in everyday activities. By investigating the development of MOT we can learn more about how children plan and execute eye, head, and body movements during activities that involve multiple moving objects, such as during team sports. By documenting a specific window of time of the typical developmental trajectory of object tracking, our results may also inform situations involving atypical development of tracking abilities by allowing parents, teachers, clinicians, and researchers to identify developmental delays early on. TrackFX could serve as a potential measure for assessing tracking ability in both typically and atypically developing children and adults.

Our study raises the critical question of whether we can improve MOT abilities in children through gameplay. Recent research with adults on the effects of playing video games suggests that we may in fact be able to increase our visual attention and tracking abilities with certain types of training (Green and Bavelier 2006b) have shown that action video-game players have greater tracking capacities than non-video-game players, and they can process information faster and extract more information in a single brief glance. These advantages were also observed in a training study after non-video-game players played a first-person, action video game for $10 \mathrm{~h}$. A separate study is needed to examine whether children could benefit from visual training using TrackFX.

Finally, and importantly, all children had fun playing the game. From the perspective of the children, TrackFX was not a cognitive task assessing their object tracking performance but a fun game in which they wanted to "catch" as many ladybugs as possible. From the perspective of our multidisciplinary team, the results from TrackFX shed light on the early visual-spatial abilities of toddlers as well as the game elements that may influence visualspatial learning. We contribute to the disciplines of developmental and cognitive psychology with a valid tool for acquiring information about changes in object tracking performance with age and with practice. With this work we also extend the state of the art of knowledge in designing and implementing a touch tablet based game to support very young children's interaction and learning.

Open Access This article is distributed under the terms of the Creative Commons Attribution License which permits any use, distribution, and reproduction in any medium, provided the original author(s) and the source are credited.

\section{References}

Alvarez, G., \& Franconeri, S. (2007). How many objects can you track?: Evidence for a resource-limited attentive tracking mechanism. Journal of Vision, 7(13),14, 1-10. 
Barker, K., Allen, R., \& McGeorge, P. (2010). Multiple-object tracking: enhanced visuospatial representations as a result of experience. Experimental Psychology, 57(3), 208-214. doi:10.1027/1618-3169/ a000026.

Beaton, E. A., Stoddard, J., Lai, S., Lackey, J., Shi, J., Ross, J. L., et al. (2010). Atypical functional brain activation during a multiple object tracking task in girls with Turner syndrome: Neurocorrelates of reduced spatiotemporal resolution. American Journal on Intellectual and Developmental Disabilities, 115(2), 140-156. doi:10.1352/1944-7558-115.2.140.

Becker, K. (2007). Digital game-based learning once removed: Teaching teachers. British Journal of Educational Technology, 38(3), 478-488.

Bowman, R. F. (1982). A "Pac-Man" theory of motivation: Tactical implications for classroom instruction. Educational Technology, 22(9), 14-16.

Couse, L. J., \& Chen, D. W. (2010). A tablet computer for young children? Exploring its viability for each childhood education. Journal of Research on Technology in Education, 43(1), 75-98.

Csikszentmihalyi, M. (1988). The flow experience and human psychology. In M. Csikszentmihalyi \& I. S. Csikszentmihalyi (Eds.), Optimal experience (pp. 15-35). Cambridge: Cambridge University Press.

Cullingford, G., Mawdesley, M. J., \& Davies, P. (1979). Some experiences with computer based games in civil engineering teaching. Computers \& Education, 3, 159-164.

De Lisi, R., \& Wolford, J. L. (2002). Improving children's mental rotation accuracy with computer game playing. Journal of Genetic Psychology, 163(3), 272-282. doi:10.1080/00221320209598683.

Dede, C. (2005). Planning for neomillennial learning styles: Implications for investment in technology and faculty. In D. Oblinger \& J. Oblinger (Eds.), Educating the Net Generation (pp. 15.11-15.22). Boulder, $\mathrm{CO}$ : Educause.

Dunbar, G., Hill, R., \& Lewis, V. (2001). Children's attentional skills and road behavior. Journal of experimental psychology: Applied, 7(3), 227-234.

Farzin, F., \& Rivera, S. M. (2010). Dynamic Object Representations in Infants with and without Fragile X Syndrome. Frontiers in Human Neuroscience, 4, 12. doi:10.3389/neuro.09.012.2010.

Franconeri, S., Jonathan, S., \& Scimeca, J. (2010). Tracking multiple objects is limited only by object spacing, not speed, time, or capacity. Psychological Science, 21, 920-925.

Gee, J. P. (2003). What video games have to teach us about learning and literacy. New York, NY: Palgrave Macmillan.

Gee, J. P. (2007). Good video games and good learning: Collected essays on video games, learning and literacy (New Literacies and Digital Epistemologies). New York, NY: Peter Lang.

Gredler, M. (2004). Games and simulations and their relationships to learning. In D. Jonassen (Ed.), Handbook of research on educational communications and technology (pp. 571-581). Mahwah, NJ: Lawrence Erlbaum Associates.

Green, C. S., \& Bavelier, D. (2003). Action video game modifies visual selective attention. Nature, 423(6939), 534-537. doi:10.1038/nature01647.

Green, C. S., \& Bavelier, D. (2006a). Effect of action video games on the spatial distribution of visuospatial attention. Journal of Experimental Psychology: Human Perception and Performance, 32(6), 1465-1478. doi:10.1037/0096-1523.32.6.1465.

Green, C. S., \& Bavelier, D. (2006b). Enumeration versus multiple object tracking: the case of action video game players. Cognition, 101(1), 217-245. doi:10.1016/j.cognition.2005.10.004.

Habgood, M. P. J., \& Overmars, M. (2006). The game maker's apprentice: Game development for beginners. Berkeley, CA: Apress.

Hahler, E. M., Tinjust, D., Mottron, L., \& Faubert, J. (2010). 3D-multiple object tracking in autism. Paper presented at the International Meeting for Autism Research, Philadelphia.

Hourcade, J. P., Bullock-Rest, N. E., \& Hansen, T. E. (2011). Multitouch tablet applications and activities to enhance the social skills of children with autism spectrum disorders. Personal and Ubiquitous Computing, 16(2), 157-168.

Kipp, K. (2005). A developmental perspective on the measurement of cognitive deficits in attention-deficit/ hyperactivity disorder. Biological Psychiatry, 57(11), 1256-1260. doi:10.1016/j.biopsych.2005.03.012.

Malone, T. W., \& Lepper, M. R. (1987). Making learning fun: A taxonomy of intrinsic motivations for learning. In R. E. Snow \& M. J. Farr (Eds.), Aptitude, learning and instruction: III. Conative and affective process analyses (pp. 223-253). Hillsdale, NJ: Erlbaum.

McClurg, P. A., \& Chaillé, C. (1987). Computer games: environments for developing spatial cognition? Journal of Educational Computing Research, 3(1), 95-111.

Nielsen (2012). American families see tablets as playmate, teacher and babysitter. Retrieved May 29, 2012, from http://blog.nielsen.com/nielsenwire/online_mobile/american-families-see-tablets-as-playmate-tea cher-and-babysitter/. 
O’Hearn, K., Hoffman, J. E., \& Landau, B. (2010). Developmental profiles for multiple object tracking and spatial memory: Typically developing preschoolers and people with Williams syndrome. Developmental Science, 13(3), 430-440. doi:10.1111/j.1467-7687.2009.00893.x.

O’Hearn, K., Lakusta, L., Schroer, E., Minshew, N., \& Luna, B. (2011). Deficits in adults with autism spectrum disorders when processing multiple objects in dynamic scenes. Autism Research, 4(2), 132-142. doi:10.1002/aur.179.

O'Hearn, K., Landau, B., \& Hoffman, J. E. (2005). Multiple object tracking in people with Williams syndrome and in normally developing children. Psychological Science, 16(11), 905-912. doi: 10.1111/j.1467-9280.2005.01635.x.

Pylyshyn, Z. W. (2000). Situating vision in the world. Trends in Cognitive Sciences, 4, 197-207.

Pylyshyn, Z. W., \& Storm, R. W. (1988). Tracking multiple independent targets: evidence for a parallel tracking mechanism. Spatial Vision, 3(3), 179-197.

Scholl, B. J. (2009). What have we learned about attention from multiple object tracking (and vice versa)? In D. Dedrick \& L. Trick (Eds.), Computation, cognition, and Pylyshyn (pp. 49-78). Cambridge, MA: MIT Press.

Subrahmanyam, K., \& Greenfield, P. M. (1994). Effect of video game practice on spatial skills in girls and boys. Journal of Applied Developmental Psychology, 15, 13-32.

Trick, L. M., Audet, D., \& Dales, L. (2003). Age differences in enumerating things that move: implications for the development of multiple-object tracking. Memory \& Cognition, 31(8), 1229-1237.

Trick, L. M., Jaspers-Fayer, F., \& Sethi, N. (2005). Multiple-object tracking in children: The "Catch the Spies" task. Cognitive Development, 20, 373-387.

Uttal, D. H., Meadow, N. G., Tipton, E., Hand, L. L., Alden, A. R., Warren, C., \& Newcombe, N. S. (2012). The malleability of spatial skills: A meta-analysis of training studies. Psychological Bulletin. doi: $10.1037 / \mathrm{a} 0028446$.

van Eck, R. (2006). Digital game-based learning: It's not just the digital natives who are restless. Educause Review, 41(2), 16-30.

Kimiko Ryokai is an assistant professor at the School of Information and Center for New Media at University of California, Berkeley. Kimiko received her PhD in Media Arts and Sciences from MIT in 2005.

Faraz Farzin is a postdoc fellow at Stanford University Department of Psychology. Faraz received her PhD in Developmental Psychology from University of California, Davis in 2010.

Eric Kaltman is a graduate student at University of California, Santa Cruz. Eric received his MA in Asian Studies in 2008 .

Greg Niemeyer is an associate professor for New Media at University of California, Berkeley. Greg received his MFA from Stanford University in New Media in 1997. 